\title{
The Clinical Value of PELP1 for Breast Cancer: A Comparison with Multiple Cancers and Analysis in Breast Cancer Subtypes
}

\author{
Xingen Wang, MMed ${ }^{1}$ \\ Julia Y. S. Tsang, $\mathrm{PhD}^{2}$ \\ Michelle A Lee, MSc ${ }^{2}$ \\ Yun-Bi Ni, PhD2 \\ Joanna H Tong, $\mathrm{PhD} \mathrm{C}^{2}$ \\ Siu-Ki Chan, FHKCP3 \\ Sai-Yin Cheung, FHKCP \\ Ka Fai To, FRCPA² \\ Gary M Tse, FRCPC 2
}

${ }^{1}$ Department of Pathology, Peking University Shenzhen Hospital, Shenzhen, China, ${ }^{2}$ Department of Anatomical and Cellular Pathology, Prince of Wales Hospital, The Chinese University of Hong Kong, Hong Kong, ${ }^{3}$ Department of Pathology, Kwong Wah Hospital, Hong Kong,

${ }^{4}$ Department of Pathology,

Tuen Mun Hosiptal, Hong Kong
Correspondence: Gary M Tse, FRCPC

Department of Anatomical and

Cellular Pathology, Prince of Wales Hospital,

The Chinese University of Hong Kong,

Ngan Shing Street, Shatin, Hong Kong

Tel: 852-35052359

Fax: 852-26374858

E-mail: garytse@cuhk.edu.hk

Received May 29, 2018

Accepted August 21, 2018

Published Online August 23, 2018

\begin{abstract}
Purpose
Proline, glutamic acid, and leucine-rich protein 1 (PELP1), a novel nuclear receptor (NR) co-regulator, is highly expressed in breast cancer. We investigated its expression in breast cancer subtypes, in comparison with other breast markers as well as cancers from different sites. Its prognostic relevance with different subtypes and other NR expression was also examined in breast cancers.
\end{abstract}

\section{Materials and Methods}

Immunohistochemical analysis was performed on totally 1,944 cancers from six different organs.

\section{Results}

PELP1 expression rate was the highest in breast cancers (70.5\%) among different cancers. Compared to GATA3, mammaglobin and gross cystic disease fluid protein 15, PELP1 was less sensitive than GATA3 for luminal cancers, but was the most sensitive for non-luminal cancers. PELP1 has low expression rate $(<20 \%)$ in colorectal cancers, gastric cancers and renal cell carcinomas, but higher in lung cancers (49.1\%) and ovarian cancers (42.3\%). In breast cancer, PELP1 expression was an independent adverse prognostic factor for nonluminal cancers (disease-free survival [DFS]: hazard ratio [HR], 1.403; $p=0.012$ and breast cancer specific survival: HR, 1.443; $p=0.015)$. Interestingly, its expression affected the prognostication of androgen receptor (AR). AR ${ }^{\text {posPELP1 }}{ }^{\text {lo }}$ luminal cancer showed the best DFS (log-rank=8.563, $p=0.036$ ) while $A R^{\text {neg } P E L P} 1^{\text {hi }}$ non-luminal cancers showed the worst DFS (log-rank=9.536, $p=0.023$ ).

\section{Conclusion}

PELP1 is a sensitive marker for breast cancer, particularly non-luminal cases. However, its considerable expression in lung and ovarian cancers may limit its utility in differential diagnosis in some scenarios. PELP1 expression was associated with poor outcome in non-luminal cancers and modified the prognostic effects of AR, suggesting the potential significance of NR co-regulator in prognostication.

\section{Introduction}

Proline, glutamic acid and leucine rich protein 1 (PELP1) also known as modulator of the non-genomic actions of estrogen receptor (ER), is a co-regulator of multiple nuclear receptors (NR), including ER, progesterone receptor (PR),
Key words

PELP1, Breast neoplasms, Differential diagnosis, Prognosis, Immunohistochemistry 
ulating gene transcription [2]. It also directly interacts with phosphoinositide 3-kinase p85 subunit and epidermal growth factor receptor (EGFR), participating in growth factor mediated enhancement of ER transactivation [3]. It possesses oncogenic potential as several of its regulated genes are involved in cancer progression and estrogen signaling [4].

A higher expression of PELP1 was found in invasive breast cancer compared to normal breast tissue or ductal carcinoma in situ [5]. Its over-expression in mouse mammary gland tissue led to early occurrence of duct hyperplasia and precancerous lesions, and promoted the development of breast cancer [6]. As an ER coregulator, PELP1 regulates estradiolmediated effects in breast cancer cells [7]. All these attested to the potential clinical utility of PELP1 in prognostication of breast cancer. Few studies examined the clinicopathologic aspects of PELP1 in breast cancers [8-10]. Cytoplasmic PELP1 expression, using a non-commercial laboratory developed antibody, was associated with higher tumor grade, Ki67 index and resistance to tamoxifen treatment [8]. Other reports using commercially available antibodies showed nuclear staining [9-11]. One study demonstrated an independent poor prognostic role of PELP1 expression [9] while a combined analysis of Ki67 and PELP1 was found to be prognostic in triple negative breast cancers (TNBC) [10].

Given its high expression in breast cancers [9], PELP1 protein may have a diagnostic potential. In TNBC, over $90 \%$ expression was observed and the immunoreactivity was consistently maintained in paired primary and metastatic TNBC cases [11]. It also showed a better performance than GATA3 in TNBCs [11]. However, currently little is known about PELP1 expression in other breast cancer subtypes and its comparison with other breast cancer markers. Additionally, aberrant expression of PELP1 in clinical cancers was examined mainly in hormonal cancers, including endometrial cancers [12], ovarian cancers [13], and prostate cancers [14]. PELP1 oncogenic signaling has been implicated in the progression of some other cancers, such as lung [15] and colon [16] cancers. To date, only few studies have evaluated the expression of PELP1 in these cancers [15].

To clarify the prognostic and diagnostic roles of PELP1 in breast cancer, a comprehensive analysis of PELP1 expression in primary tumor from breast, lung, colorectal, kidney, ovary, and stomach using tissue microarrays (TMA) and immunohistochemistry was performed. In addition, PELP1 was compared to other breast cancer biomarkers in different breast cancer subtypes. The prognostic impact of PELP1 in different breast cancer subtypes and interactions with other NR were also examined.

\section{Materials and Methods}

\section{Patients data}

Consecutive paraffin embedded samples from breast cancer patients over a period of 4 (2002-2005), 7 (2003-2009), and 4 (2003-2006) years were obtained from the three involved institutions for TMA construction. Archival cohorts of lung carcinoma, ovary carcinoma, colorectal carcinoma, gastric carcinoma, and renal cell carcinoma (RCC) were also included. All the specimens were routinely processed and stained with hematoxylin and eosin (H\&E). For breast cancer, the diagnosis was confirmed according to World Health Organization criteria and graded $[17,18]$. Lymphovascular invasion (LVI) and fibrotic focus (FF) were evaluated as previously described [19]. Patients' age, pN category, pT category, and outcome data were retrieved from the medical records. Breast cancer specific survival (BCSS) was defined as the time interval from the date of initial diagnosis to the date of breast cancer related death. Disease-free survival (DFS) was defined as the time interval from the date of initial diagnosis to the first detection of breast cancer specific relapse or death. For pulmonary cancers, EGFR mutation and smoking status were retrieved from previous database. For other cancers, patients' information, including age, sex, histologic diagnosis, TNM stage, and tumor size were retrieved from medical records.

\section{TMA construction and immunohistochemistry}

TMA containing representative tumor areas were constructed with duplicated 0.6-mm cores as previously described [20]. One section from each TMA was stained with H\&E and reviewed to confirm the presence of representative tumors. Immunohistochemical (IHC) staining of PELP1 (1:500, polyclonal antibody, Bethyl Laboratories Inc., Montgomery, TX) was performed using Ultraview Universal DAB Detection Kit (Ventana, Tucson, AZ) after deparaffinization, rehydration, and antigen retrieval of the slides. All slides were counterstained with hematoxylin. The tumor cells in the TMA slides were assessed for the staining intensity, and the actual percentage of stained cells in the nucleus by two of the authors blinded to the clinical information and the staining results of other markers. The percentage of cells with moderate/strong nuclear staining was recorded. For its diagnostic role, PELP1 staining was classified into low $(<5 \%)$, focal $(5 \%-49 \%)$, and diffuse $(\geq 50 \%)$. A cutoff of $5 \%$ was used to define PELP1 positive expression in a case (i.e., focal/ diffuse PELP1 expression). The expression rate of PELP1 was determined as the percentage of cases with PELP1 positivity. For survival analysis, the median value of proportion of PELP1 

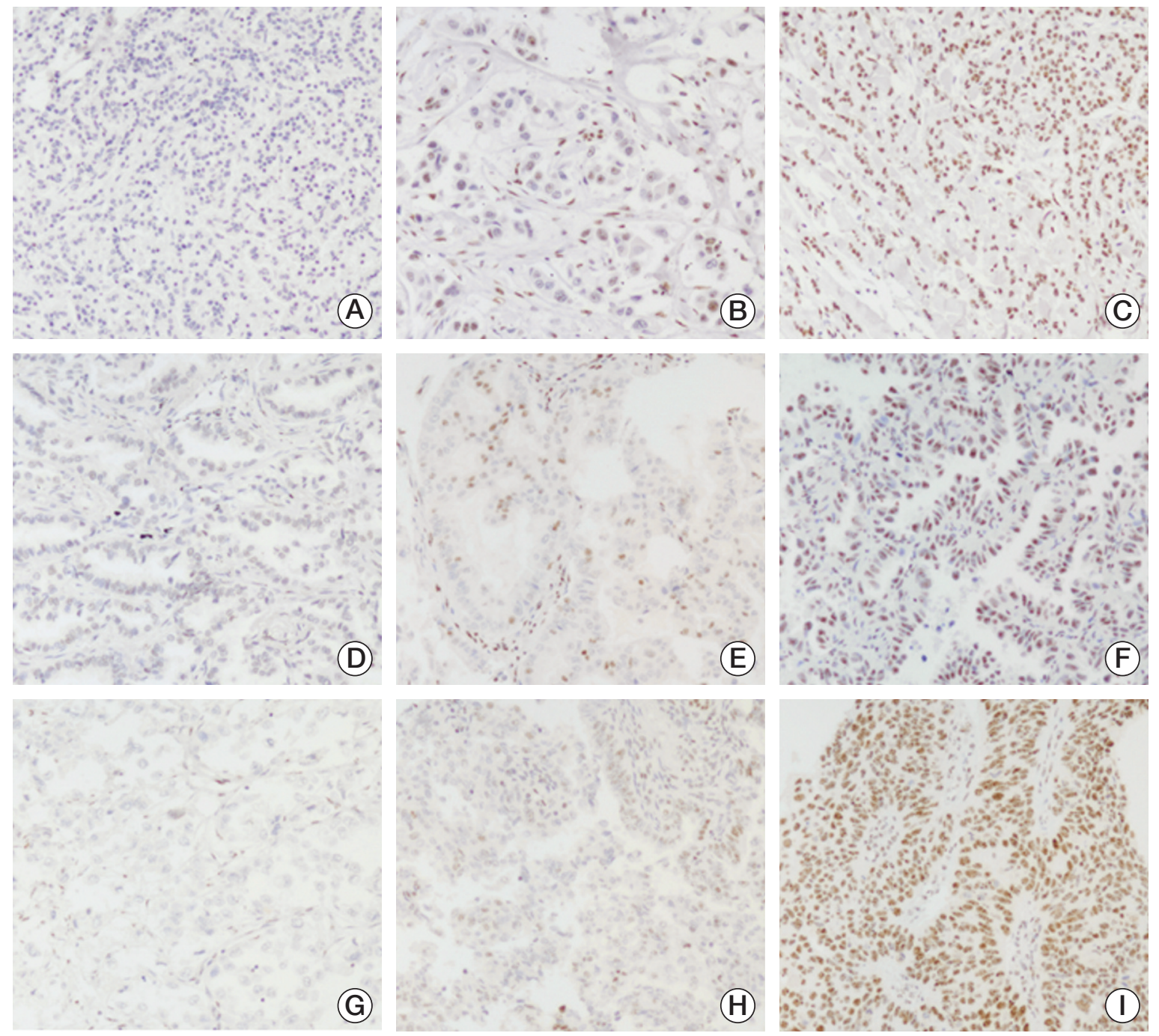

Fig. 1. Representative staining of proline, glutamic acid, and leucine-rich protein 1 expression on breast cancers (A, low; $B$, focal; C, diffuse staining), lung cancers (D, low; E, focal; F, diffuse staining), and ovarian cancers (G, low; H, focal; I, diffuse staining) $(\times 200)$.

stained cells in a case (i.e., 70\%) was used to classify the cases into two groups, i.e., PELP1 $1^{\text {lo }}$ ( $<70 \%$ PELPL1 stained cells) and PELP1 ${ }^{\text {hi }}(\geq 70 \%$ PELP1 stained cells) groups. Data on other biomarkers for the breast cancer cohort including NR (ER, PR, and AR), Ki67, growth factor receptors (human epidermal growth factor receptor 2 [HER2], EGFR), basal cytokeratins (CK5/6 and CK14), and other breast diagnostic markers (GATA3, gross cystic disease fluid protein 15 [GCDFP15], and mammaglobin [MGB]) expression were retrieved from our database (details on IHC staining were shown in S1 Table). IHC surrogate was used for molecular subtyping, defined as luminal A (ER/ and PR+, HER2-, Ki67 $<20 \%, \mathrm{PR} \geq 20 \%$ ), luminal B (ER/and PR+, HER2+ / and Ki67
$>20 \%$ / and PR < 20\%), HER2-overexpressed (HER2-OE) (ER-, PR-, HER2+), basal-like breast cancers (BLBC) (ER-, PR-, HER2-, CK5 / 6+ / and EGFR+), and unclassified (quintuple negative [5NP]) (ER-, PR-, HER2-, CK5/6-, EGFR-) $[21,22]$.

\section{Statistical analysis}

The findings were analyzed using the statistical software SPSS for Windows ver. 23 (IBM Corp., Armonk, NY). Chisquare analysis or Fisher exact test was used to test for the association of PELP1 expression with tumor grade and biomarkers. Survival data were evaluated with Kaplan-Meier 
Table 1. Expression of PELP1 in different tumors

\begin{tabular}{|c|c|c|c|c|c|c|}
\hline & \multicolumn{2}{|c|}{$\begin{array}{c}\text { Proportion of } \\
\text { PELPL1+ve cells }\end{array}$} & \multicolumn{4}{|c|}{ No. of cases } \\
\hline & Mean & Median & Low & Focal & Diffuse & Total \\
\hline Breast $^{\text {a) }}$ & 51.9 & 70 & 317 (29.5) & $21(2.0)$ & 735 (68.5) & 1,073 \\
\hline Lunga) & 34.8 & 0 & $160(50.8)$ & $9(2.9)$ & $146(46.3)$ & 315 \\
\hline Ovary ${ }^{a)}$ & 32.4 & 0 & 64 (57.7) & $1(0.9)$ & $46(41.4)$ & 111 \\
\hline Colorectal & 8.7 & 0 & $179(86.5)$ & $3(1.4)$ & $25(12.1)$ & 207 \\
\hline Gastric & 2.3 & 0 & $124(94.7)$ & $5(3.8)$ & $2(1.5)$ & 131 \\
\hline RCC & 7.9 & 0 & $84(85.7)$ & $6(6.1)$ & $8(8.1)$ & 98 \\
\hline
\end{tabular}

Values are presented as number (\%). PELP1, proline, glutamic acid, and leucine-rich protein 1; RCC, renal cell carcinoma.

a) A statistical significant difference $(\mathrm{p}<0.001)$ was found in the expression rate between breast, lung, and ovarian cancers.

analysis with a log rank test. Multivariate Cox hazard regression analysis was used to evaluate any independent prognostic effect of the variables. Statistical significance was established at $\mathrm{p}<0.05$.

\section{Ethical statement}

The study was approved by Joint Chinese University of Hong Kong-New Territories East Cluster clinical research ethics committee with a waiver of informed consent (IRB No. 2011.199) and performed in accordance with the principles of the Declaration of Helsinki.

\section{Results}

\section{The expression of PELP1 in different tumors}

Totally 1,073 invasive breast cancers, 325 lung cancers, 111 ovarian cancers, 207 colorectal cancers, 131 gastric cancers, and 98 RCC were included for assessment (details on the clinicopathological characteristics of the cohort were shown in S2 Table; no clinical data were available for RCC).

PELP1 staining was nuclear, with no cytoplasmic staining in all tumor types. Among the different cancers, PELP1 showed the highest expression rate in breast cancers $(70.5 \%$ of cases with focal/ diffuse staining). Most cases (68.5\%) with PELP1 expression showed diffuse staining and 21 cases $(2.0 \%)$ showed focal staining. The second highest expression rate was found in lung cancers $(46.3 \%$ and $2.9 \%$ of cases with diffuse and focal staining respectively), followed by ovarian cancers $(41.1 \%$ and $0.9 \%$ of cases with diffuse and focal stainin, respectively). There was a statistical significance in the expression rate among these three cancer types $(\mathrm{p}<0.001)$ (representative staining was shown in Fig. 1). Less than 15\% of colorectal cancer, RCC and gastric cancers showed focal/ diffuse PELP1 expression (Table 1).

In lung and ovarian cancers, PELP1 expression rate correlated with clinicopathological features. High PELP1 expression rate was associated with non-adenocarcinoma, EGFR wild type (WT) cancers and smoking history (both current and ex-smokers) in lung cancers ( $p<0.001$ for all). Its association with EGFR WT status was also observed in lung adenocarcinomas ( $p<0.001)$ (S3 Table). In ovarian cancers, PELP1 expression correlated with small tumor size $(\mathrm{p}=0.023)$. However, there were no correlation with histological types, International Federation of Gynecology and Obstetrics staging, laterality and age (S4 Table).

\section{The expression of PELP1 in subgroups of breast cancers}

In breast cancer, increased PELP1 expression rate was associated with the absence of FF $(\mathrm{p}=0.001)$ and younger patients' age ( $\mathrm{p}=0.007)$. Among the 1,063 breast cancers with complete IHC data for molecular classification, a significant differential distribution of PELP1 expression was found, with diffuse staining mostly in luminal B $(76.4 \%, 246 / 322)$, followed by BLBC $(72.7 \%, 48 / 66)$, HER2-OE $(68.9 \%, 73 / 106)$, luminal A $(63.7 \%, 310 / 487)$, and 5NP $(58.5 \%, 48 / 82)$ subtypes (Table 2) $(p=0.012)$. Given the relationship of PELP1 with NR, growth factor receptor signaling and epithelialmesenchymal transition (EMT) processes, correlation analysis of PELP1 were performed with biomarkers relating to these pathways. Its associations with other breast markers were also examined. PELP1 expression rate was found to be positively associated with AR, Ki-67, HER2, EGFR, p-cadherin, and GATA-3 $(\mathrm{p} \leq 0.045)$ (Table 2). 
Table 2. Clinicopathological association of PELP1 expression in breast cancers

\begin{tabular}{|c|c|c|c|c|c|}
\hline \multirow{2}{*}{$\begin{array}{l}\text { Clincopathological } \\
\text { feature }\end{array}$} & \multicolumn{3}{|c|}{ PELP1 } & \multirow{2}{*}{ Total } & \multirow{2}{*}{ p-value } \\
\hline & Low & Focal & Diffuse & & \\
\hline \multicolumn{6}{|l|}{ Grade } \\
\hline 1 & $46(28.4)$ & $6(3.7)$ & $110(67.9)$ & 162 & 0.903 \\
\hline 2 & $127(30.1)$ & $8(18.9)$ & $287(68.0)$ & 422 & \\
\hline 3 & $144(29.5)$ & $7(1.4)$ & $338(69.1)$ & 489 & \\
\hline \multicolumn{6}{|l|}{ LVI } \\
\hline Absent & $216(29.4)$ & $15(2.0)$ & $504(68.6)$ & 735 & 0.723 \\
\hline Present & $88(30.7)$ & $5(1.7)$ & $194(67.6)$ & 287 & \\
\hline \multicolumn{6}{|l|}{ FF } \\
\hline Absent & $216(27.3)$ & $14(17.7)$ & $561(70.9)$ & 791 & 0.001 \\
\hline Present & $98(37.4)$ & $7(26.7)$ & 157 (29.9) & 262 & \\
\hline \multicolumn{6}{|l|}{$\mathrm{pT}$} \\
\hline 1 & $116(27.0)$ & $11(2.6)$ & $303(70.4)$ & 430 & 0.125 \\
\hline 2 & $174(31.1)$ & $9(1.6)$ & $376(67.3)$ & 559 & \\
\hline 3 & 19 (34.5) & 0 & $36(65.5)$ & 55 & \\
\hline 4 & $6(37.5)$ & 0 & $10(62.5)$ & 16 & \\
\hline \multicolumn{6}{|l|}{$\mathrm{pN}$} \\
\hline 0 & $149(28.4)$ & $11(2.1)$ & $364(69.5)$ & 524 & 0.309 \\
\hline 1 & $94(30.9)$ & $5(1.7)$ & $205(67.4)$ & 304 & \\
\hline 2 & $36(27.9)$ & $1(0.8)$ & $92(71.3)$ & 129 & \\
\hline 3 & $31(36.1)$ & $2(2.3)$ & $53(61.6)$ & 86 & \\
\hline \multicolumn{6}{|l|}{ Molecular subtype } \\
\hline Luminal A & $166(34.1)$ & $11(2.3)$ & $310(63.6)$ & 487 & 0.012 \\
\hline Luminal B & $72(22.4)$ & $4(1.2)$ & $246(76.4)$ & 322 & \\
\hline HER2-OE & $31(29.2)$ & $2(1.9)$ & 73 (68.9) & 106 & \\
\hline BLBC & $16(24.3)$ & $2(3.0)$ & $48(72.7)$ & 66 & \\
\hline $5 \mathrm{NP}$ & $32(39.0)$ & $2(2.5)$ & $48(58.5)$ & 82 & \\
\hline \multicolumn{6}{|l|}{ Age (yr) } \\
\hline Mean \pm SD & $56.4 \pm 13.1$ & $57.0 \pm 14.5$ & $53.8 \pm 12.4$ & $54.7 \pm 12.7$ & 0.007 \\
\hline Range & 28-97 & 35-94 & 22-91 & & \\
\hline \multicolumn{6}{|l|}{ Size $(\mathrm{cm})$} \\
\hline Mean \pm SD & $2.75 \pm 1.43$ & $2.27 \pm 1.10$ & $2.66 \pm 1.48$ & $2.68 \pm 4.46$ & 0.204 \\
\hline Range & $0.2-10.1$ & $0.8-4.2$ & $0.2-13.0$ & & \\
\hline \multicolumn{6}{|l|}{ ER } \\
\hline Negative & $95(30.1)$ & $9(2.8)$ & $212(67.1)$ & 316 & 0.702 \\
\hline Positive & $222(29.5)$ & $12(1.6)$ & $518(68.9)$ & 752 & \\
\hline \multicolumn{6}{|l|}{ PR } \\
\hline Negative & $114(31.7)$ & $7(1.9)$ & $239(66.4)$ & 360 & 0.318 \\
\hline Positive & $202(28.7)$ & $14(2.0)$ & 488 (69.3) & 704 & \\
\hline \multicolumn{6}{|l|}{ AR } \\
\hline Negative & $221(39.9)$ & $12(2.2)$ & 321 (57.9) & 554 & $<0.001$ \\
\hline Positive & 93 (18.5) & $9(1.8)$ & $400(79.7)$ & 502 & \\
\hline \multicolumn{6}{|l|}{ Ki67 } \\
\hline Low & $266(34.1)$ & $16(2.1)$ & 497 (63.8) & 779 & $<0.001$ \\
\hline High & 48 (17.1) & $5(1.8)$ & $228(81.1)$ & 281 & \\
\hline \multicolumn{6}{|l|}{ HER2 } \\
\hline Negative & $267(31.1)$ & $17(2.0)$ & 575 (66.9) & 859 & 0.045 \\
\hline Positive & $50(24.0)$ & $4(1.9)$ & $154(74.1)$ & 208 & \\
\hline
\end{tabular}

(Continued to the next page) 
Table 2. Continued

\begin{tabular}{|c|c|c|c|c|c|}
\hline \multirow{2}{*}{$\begin{array}{l}\text { Clincopathological } \\
\text { feature }\end{array}$} & \multicolumn{3}{|c|}{ PELP1 } & \multirow{2}{*}{ Total } & \multirow{2}{*}{ p-value } \\
\hline & Low & Focal & Diffuse & & \\
\hline \multicolumn{6}{|l|}{ EGFR } \\
\hline Negative & $306(30.6)$ & $21(2.1)$ & $674(67.3)$ & 1,001 & 0.001 \\
\hline Positive & $6(11.1)$ & 0 & $48(88.9)$ & 54 & \\
\hline \multicolumn{6}{|l|}{ CK5/6 } \\
\hline Negative & 295 (30.9) & $18(1.9)$ & $641(67.2)$ & 954 & 0.015 \\
\hline Positive & 21 (19.3) & $3(2.7)$ & $85(78.0)$ & 109 & \\
\hline \multicolumn{6}{|l|}{ CK14 } \\
\hline Negative & $305(30.6)$ & $19(1.9)$ & $673(67.5)$ & 997 & 0.017 \\
\hline Positive & 10 (15.9) & $2(3.2)$ & $51(80.9)$ & 63 & \\
\hline \multicolumn{6}{|l|}{ Vimentin } \\
\hline Negative & $281(30.8)$ & $17(1.9)$ & $615(67.3)$ & 913 & 0.070 \\
\hline Positive & 33 (22.9) & $4(2.8)$ & $107(74.3)$ & 144 & \\
\hline \multicolumn{6}{|l|}{ P-cadherin } \\
\hline Negative & $265(32.9)$ & $12(1.5)$ & $528(65.6)$ & 805 & 0.001 \\
\hline Positive & $50(20.7)$ & $8(3.3)$ & $184(76.0)$ & 242 & \\
\hline \multicolumn{6}{|l|}{ GATA3 } \\
\hline Low & $70(38.0)$ & $4(2.2)$ & $110(59.8)$ & 184 & 0.012 \\
\hline Focal/Diffuse & $243(28.8)$ & $16(1.9)$ & $586(69.3)$ & 845 & \\
\hline \multicolumn{6}{|l|}{ MGB } \\
\hline Low & $96(24.1)$ & $7(1.8)$ & $295(74.1)$ & 398 & 0.302 \\
\hline Focal/Diffuse & $75(24.5)$ & $6(2.0)$ & $225(73.5)$ & 306 & \\
\hline \multicolumn{6}{|l|}{ GCDFP-15 } \\
\hline Low & $226(30.1)$ & $12(1.6)$ & $514(68.3)$ & 752 & 0.763 \\
\hline Focal/Diffuse & $84(28.5)$ & $8(2.7)$ & $203(68.8)$ & 295 & \\
\hline
\end{tabular}

Values are presented as number (\%). PELP1, proline, glutamic acid, and leucine-rich protein 1; LVI, lymphovascular invasion; $\mathrm{FF}$, fibrotic focus; HER2-OE, human epidermal growth factor receptor 2 overexpression; BLBC, basal like breast cancer; 5NP, quintuple negative; $\mathrm{SD}$, standard deviation; ER, estrogen receptor; PR, progesterone receptor; $\mathrm{AR}$, androgen receptor; EGFR, epidermal growth factor receptor; CK, cytokeratin; MGB, mammaglobin; GCDFP-15, gross cystic disease fluid protein 15.

\section{Comparison of PELP1 to other breast markers (GCDF- P15, MGB, and GATA3) in breast cancers}

In breast cancers, PELP1, GCDFP15, and GATA3 showed differential expression rates among different molecular subtypes ( $\mathrm{p} \leq 0.012$ ), but MGB did not. GCDFP15 and MGB showed relatively low expression rate $(<50 \%)$ overall and in different subtypes. GATA3 $(82.4 \%)$ showed the highest overall expression rate, followed by PELP1 $(70.5 \%)$ overall. Comparing GATA3 and PELP1 in luminal cancers, GATA3 (95.1\% and $91.6 \%$ in luminal A and B subtypes, respectively) showed a higher expression rate than PELP1 $(66.0 \%$ and $77.63 \%$ in luminal A and B subtypes, respectively). In contrast, in the non-luminal subtypes, PELP1 showed a higher expression than GATA3. In HER2-OE and TNBC, PELP1 expression rate was $70.8 \%$ and $67.4 \%$, respectively, whereas GATA3 expression rate was $60.4 \%$ and $37.5 \%$, respectively.
Moreover, a higher percentage of cases with diffuse staining was found in PELP1 positive than GATA3 positive nonluminal cases (Table 3 ). In addition, a significantly higher number of cases with PELP1 diffuse staining was found in different breast cancer subtypes compared to lung/ovarian cancers (S5 Table). PELP1 and GATA3 expression was also examined on whole sections of 15 randomly selected cases with low/diffuse staining. All diffuse cases retained PELP-1 positivity in whole section. For PELP1 low cases, 70\% remained low expression and 30\% showed focal staining. A combined expression rate of GATA3 and PELP1 to detect luminal subtypes showed less than $6 \%$ improvement compared to GATA3 alone. However, for non-luminal subtypes, their combined expression was found in $87.4 \%$ and $77.8 \%$ of HER2-OE and TNBC, respectively. Using GATA3 only the expression rates were $60.4 \%$ and $37.5 \%$ in HER2-OE and TNBC respectively; using PELP1 only, the expression rates 


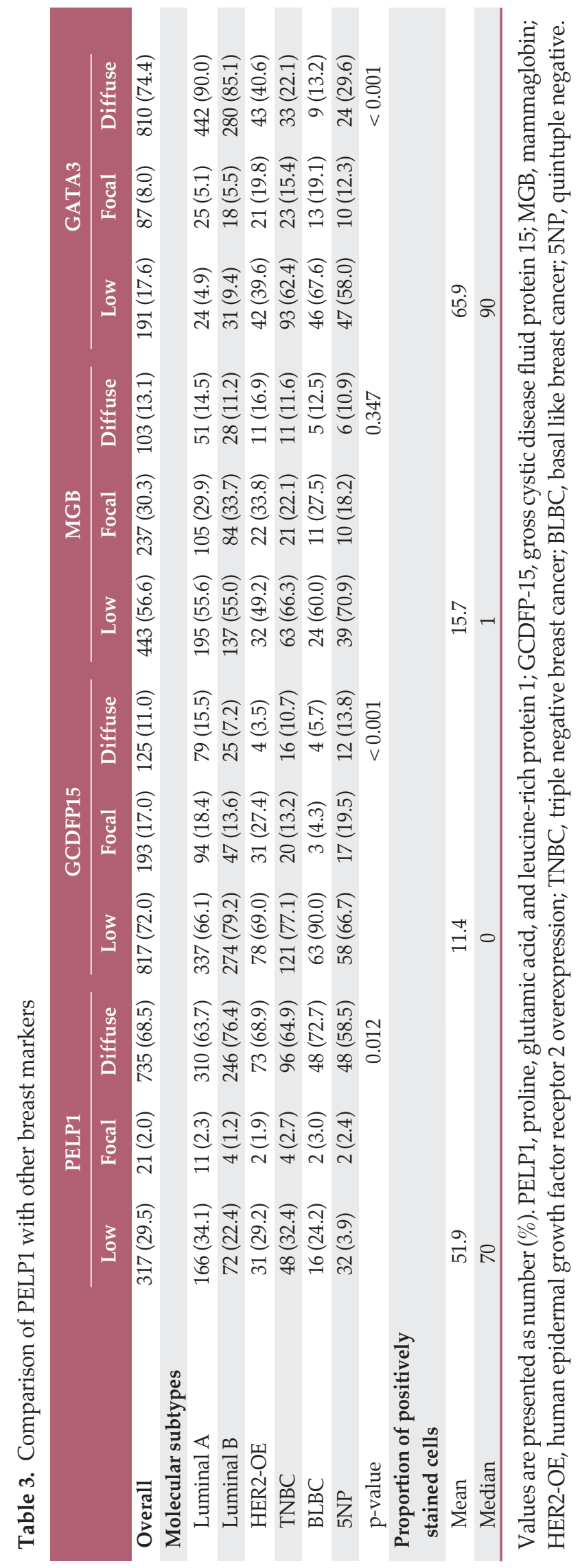

were $70.8 \%$ and $70.6 \%$ for HER2-OE and TNBC respectively. Of note, in the non-luminal subtypes, the combined expression rate of MGB and GATA3 (77.0\% and 63.3\% for HER2$\mathrm{OE}$ and TNBC respectively) was similar to PELP1 alone (S6 Table).

\section{Relationship of PELP1 with breast cancer patient's out- come}

Follow-up data was available in 940 patients, with mean follow-up time of 62.7 months (range, 1 to 210 months). Relapse or death occurred in 146 patients (15.5\%). Among them, 106 patients died of breast cancer. For survival analysis, the median proportion of PELP1 positively stained cells (i.e., 70\% of moderate and / or intensely stained cells) was used to classify PELP1 high (PELP1 ${ }^{\text {hi }}$, with $\geq 70 \%$ PELP1 positively stained cells) and PELP1 low (PELP1 ${ }^{\text {lo }}$, with $<70 \%$ PELP1 positively stained cells) subgroups. In all cases and the luminal cancers, PELP1 subgroup was not associated with patients' survival (Fig. 1). However, in the non-luminal group, PELP1 ${ }^{\text {hi }}$ cases showed shorter DFS (log-rank=3.894, $\mathrm{p}=0.048$ ) (Fig. 2). $P E L P 11^{\text {hi }}$ cases also showed a trend towards worse BCSS (log-rank=3.446, $\mathrm{p}=0.063$ ). Multivariate cox hazard analysis including age, grade, $\mathrm{pT}$, $\mathrm{pN}$ category, LVI, HER2, ER, PR, AR, and PELP1 expression demonstrated that PELP1 ${ }^{\text {hi }}$ was an independent adverse factor of DFS (hazard ratio [HR], 1.403; $\mathrm{p}=0.012 ; 95 \%$ confidence interval [CI], 1.079 to 1.851) and BCSS (HR, 1.443; $\mathrm{p}=0.015 ; 95 \% \mathrm{CI}, 1.075$ to 1.937) in the non-luminal group (Table 4).

We previously demonstrated AR to be a favorable prognostic factor in luminal cancers [20]. Considering the biological important interaction of PELP1 with NR and the correlation between the two markers in this study, we further examined the prognostic interaction of PELP1 and AR. We found that AR was associated with better DFS only in luminal cases that were $P E L P 1{ }^{10}$ (DFS: chi-square $=9.383, p=0.002$ ). In contrast, in the non-luminal cases, AR demonstrated a trend of better survival in the PELP 1 hi cases (DFS: chi-square= 3.311, $\mathrm{p}=0.069$ ). Combined analysis of AR and PEL-P1 status showed that, in luminal cancers, AR ${ }^{\text {pos PELP1 }}{ }^{\text {lo }}$ (but not $\mathrm{AR}^{\mathrm{pos} P E L P} \mathrm{1}^{\text {hi }}$ ) cases demonstrated better DFS than $\mathrm{AR}^{\text {neg }}$ PELP1 ${ }^{\text {lo }}$ cases (AR ${ }^{\text {pos }} P E L P 1^{\text {lo: }}$ log-rank=9.383, $\mathrm{p}=0.002$; $A R^{\text {posPELP1 }}{ }^{\text {hi: }}$ log-rank=1.955, $p=0.162$ ) (Fig. 3). Interestingly, among the non-luminal cancers, $A R^{\text {neg } P E L P} 1^{\text {hi }}$ cases, but not $\mathrm{AR}^{\text {pos PELP1 }}{ }^{\text {hi }}$ cases, showed the worst DFS (compared to AR $^{\text {pos} P E L P 1 ~}{ }^{\text {lo: }}$ log-rank=4.310, $\mathrm{p}=0.038$; AR $^{\text {pos }} P E L P 1^{\text {hi }}$ : logrank=3.311, $\mathrm{p}=0.069 ; \mathrm{AR}^{\text {neg }} P E L P 1^{10}$ : log-rank=7.087, $\mathrm{p}=0.008$ ) (Fig. 3). Multivariate analysis demonstrated that $\mathrm{AR}^{\text {neg- }}$ PELP1 ${ }^{\text {hi }}$ group remained to have independent prognostic value for both DFS and BCSS in non-luminal cancers (DFS: HR, 2.581; $\mathrm{p}=0.002$; BCSS: HR, 2.949; $\mathrm{p}=0.002)$ (S7 Table). 


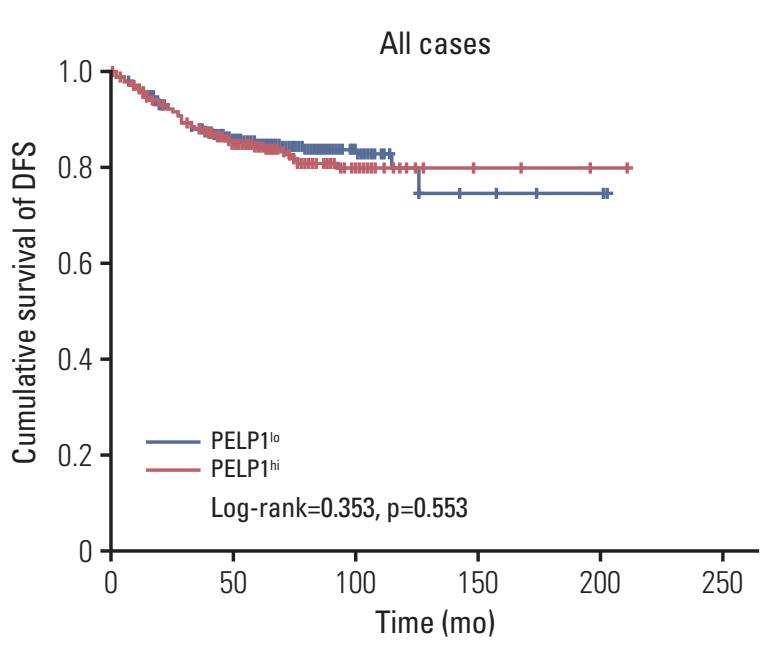

A
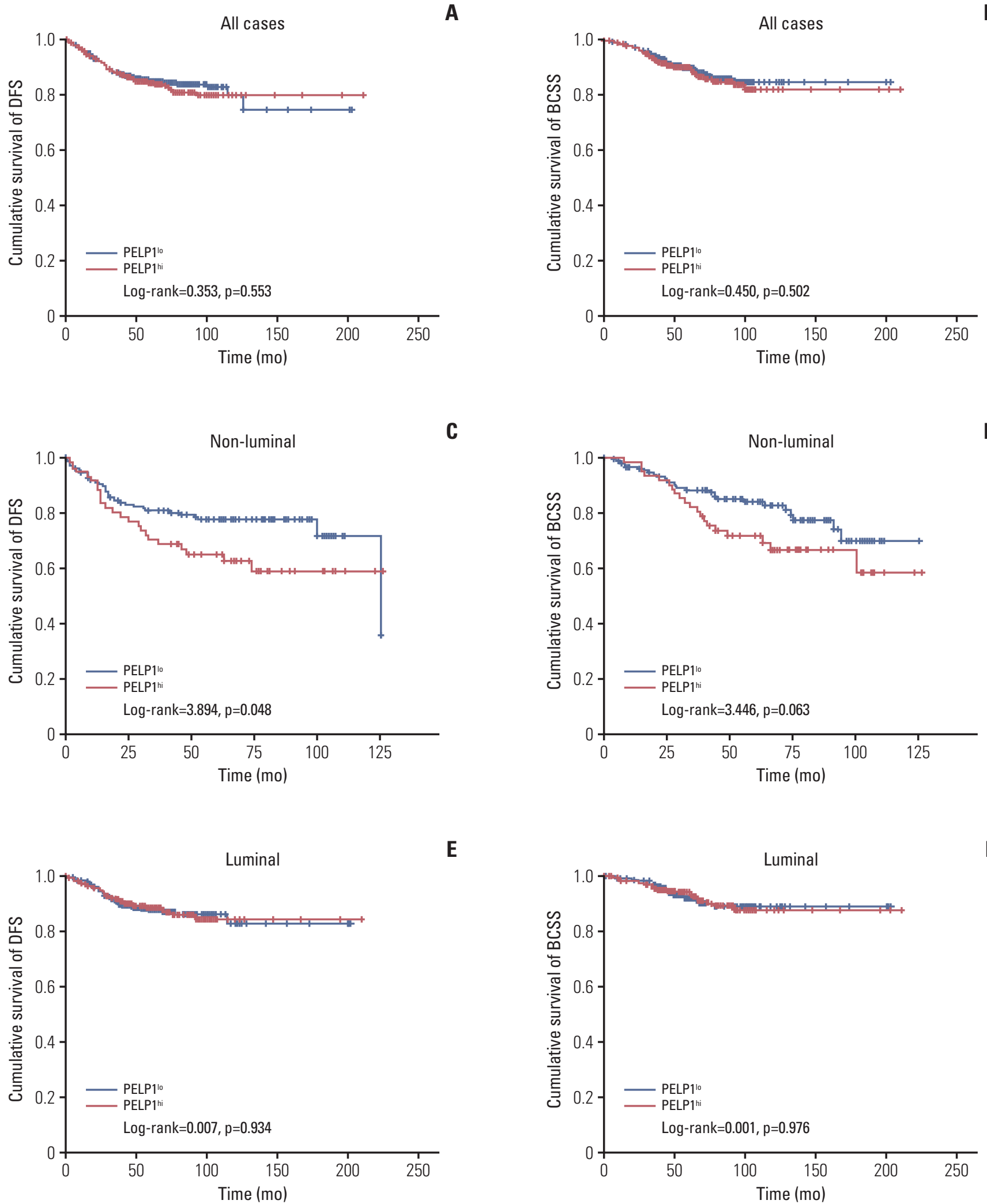

Fig. 2. Kaplan-Meier analysis of proline, glutamic acid, and leucine-rich protein 1 (PELP1) expression for disease-free survival (DFS) (A, C, E) and breast cancer specific survival (BCSS) (B, D, F) in all cases (A, B), non-luminal (C, D), and luminal (E, F) breast cancer subgroups. PELP1 ${ }^{\text {lo }}$, PELP1 low; PELPhi, PELP1 high. 
Table 4. Multivariate cox regression analysis for DFS and BCSS in non-luminal subgroup

\begin{tabular}{lccr} 
& HR & $95 \%$ CI for HR & p-value \\
\hline DFS & & & \\
Age & 0.974 & $0.952-0.997$ & 0.025 \\
pT & 1.923 & $1.322-2.796$ & 0.001 \\
pN & 1.984 & $1.544-2.550$ & $<0.001$ \\
PELP1 status & 1.403 & $1.079-1.851$ & 0.012 \\
BCSS & & & 0.023 \\
Age & 0.970 & $0.946-0.996$ & $<0.001$ \\
pT & 2.669 & $1.713-4.159$ & $<0.001$ \\
pN & 1.717 & $1.305-2.259$ & 0.015 \\
\hline PELP1 status & 1.443 & $1.075-1.937$ & \\
\hline
\end{tabular}

Backwald method was used for the analysis. Factors included in the initial steps included age, grade, LVI, pT, pN, Ki67, HER2, AR, and PELP1 status. LVI, Ki67, HER2, AR and PELP1 were analyzed as categorical variables. The absence of LVI, Ki67 low, HER2 negativity, AR negativity, and PELP1 low status were used as references in their categories. DFS, disease free survival; BCSS, breast cancer specific survival; HR, hazard ratio; CI, confidence interval; PELP1, proline, glutamic acid, and leucine-rich protein 1; LVI, lymphovascular invasion; HER2, human epidermal growth factor receptor 2; AR, androgen receptor.

\section{Discussion}

Recent studies showed a high expression rate of PELP1 in breast cancers [9] and its superiority over GATA3 in identifying TNBC [11]. These findings have prompted us to further evaluate PELP1 expression in different breast cancer subtypes with a large series of breast cancers and perform a thorough comparison with other breast markers and in other cancer types. PELP1 showed an overall expression rate of $70.5 \%$ in breast cancer. Although there was a significant differential expression in different subtypes (being most abundant in luminal B cases, $77.6 \%$ ), the expression rate in other subtype was comparable (range, $61 \%$ to $75.8 \%$ ). It was not as good as GATA3 for luminal cancers, but was better than GATA3 for non-luminal cancers. Overall, PELP1 was better than GCDFP15 and MGB. In TNBC, similar to other reported findings [11], PELP1 showed a higher sensitivity than GATA3 ( $68.4 \%$ vs. $38.4 \%)$, and this was equaled by using GATA3 and MGB together (63.3\%). Moreover, the majority of the PELP1 positive TNBC showed diffuse staining, making observation of the staining easy. The data suggests a potential use of PELP1 for metastatic TNBC in appropriate settings, such as history of primary TNBC, particularly those not expressing other breast markers (GATA3, MGB, and GCDFP15).

For its application in differential diagnosis, it is important to understand its expression pattern in cancers of diverse origins. PELP1 expression rate was mostly demonstrated in hormone-related cancers [9,12-14,23]; and for other cancers, such as RCC, colorectal cancers and lung cancers, investigators have evaluated mostly its cellular functions but not on its clinical expression $[15,16,24,25]$. Here, PELP1 expression was also evaluated in five other primary cancers. Similar to reported findings [13,23], we found a high PELP1 expression rate in ovarian cancers $(41.4 \%)$, and lower expression rates in gastric cancers $(1.5 \%)$, colorectal cancers $(13.5 \%)$, and RCC (7.9\%). PELP1 was also highly expressed in lung cancers $(68.5 \%)$, particularly non-adenocarcinoma lung cancers $(70.2 \%)$ (including squamous cell carcinoma and miscellaneous subtypes) and lung cancers with EGFR WT (57.6\%). As one of the most common metastatic sites for breast cancer is lung; and as both breast and lung cancers preferentially metastasize to the bone, liver, brain, and adrenal glands, PELP1 may not be able to reliably distinguish a primary lung from metastatic breast cancer in the lung. A panel of markers, including thyroid transcription factor 1 and other breast markers (such as a combination of GATA3 and MGB which demonstrated similar sensitivity as PELP1 also in non-luminal cancers), and detailed clinical history is crucial when differentiating these cancers.

As a prognostic marker, PELP1 was associated with poor outcome in luminal cancers [9] and in TNBC when combined with Ki67 [10]. We found an adverse prognostic significance of PELP1 in non-luminal cancers, but not in luminal cancers. In a previous study, outcome based cutoff point optimization was applied with a longer follow up time [9]. As the recurrence in luminal cancers may occur beyond 10 years [26], the mean follow-up of 62.7 months in the current cohort might not fully capture all recurrences. Nevertheless, we demon- 


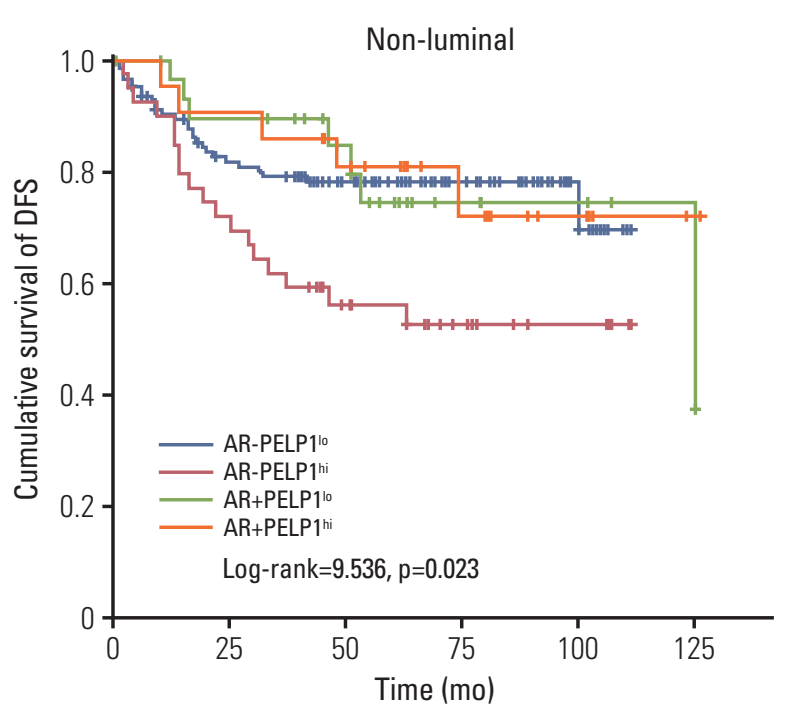

A
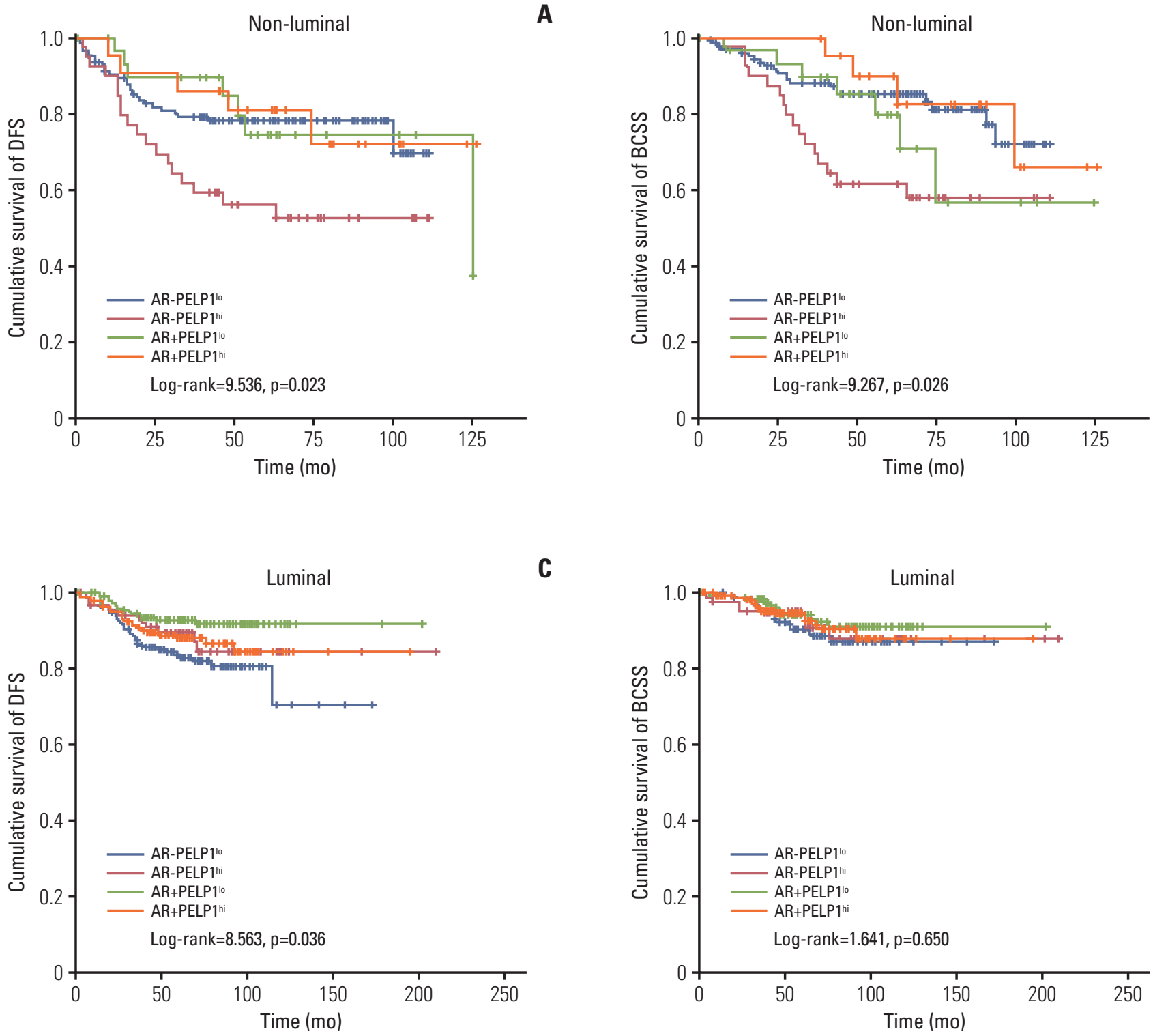

Fig. 3. Kaplan-Meier analysis for disease-free survival (DFS) (A, C) and breast cancer specific survival (BCSS) (B, D) according to proline, glutamic acid, and leucine-rich protein 1 (PELP1) and androgen receptor (AR) expression level in non-luminal (A, B) and luminal (C, D) subsets. PELP1 ${ }^{\text {lo }}$, PELP1 low; PELPhi, PELP1 high.

strated PELP1 was associated with luminal B cancers, the luminal subtype with poor prognosis. Furthermore, we observed an association of AR and PELP1 in prognostication and potentially, the underlying mechanism in different breast cancer groups. AR has been described as a favorable marker in luminal breast cancers [20]. However, reports on $\mathrm{AR}$ expression in non-luminal cancers were less consistent [27]. NR interacting co-regulators may play important roles. Interestingly, we found the best DFS in AR ${ }^{\text {posPELP1 }} 1^{\text {lo }}$ luminal cancer while the worst was in AR ${ }^{\text {neg } P E L P 1 ~}{ }^{\text {hi }}$ non-luminal can- cers. In luminal cancers, AR could compete for ER binding sites, thereby blocking E2-mediated transcription of proliferative genes [28]. The presence of PELP1 may alter such interactions. A similar phenomenon was seen in prostatic cancers, where AR and ER $\beta$ form a protein complex in the presence of PELP1 to mediate E2-mediated transcription of AR-target gene [29]. Interactions between PELP1, AR and FHL2 enhanced AR transactivation in prostate cancer [14]. In non-luminal AR breast cancers, PELP1 may possibly promote AR transactivation. Independent of NR, PELP1 can pro- 
mote cancer progression by chromatin remodeling [30]. This could occur particularly in the AR ${ }^{\text {neg }}$ non-luminal cases. In fact, PELP1 knockdown in $E^{\text {neg }} A R^{\text {neg }}$ breast cancer cell lines promoted inhibitory histone mark H3K9me2 [31]. Nonetheless, the precise molecular interaction between $A R, E R$, and PELP1 in breast cancer remains to be determined and warrants further analysis.

Apart from its interaction with AR, PELP1 was associated positively with a number of biomarkers, including Ki-67, HER2, EGFR, and basal markers. These associations reflected the critical functions of PELP1 in several pathways related to these markers. PELP1 may enhance breast cancer proliferation by increasing local estrogen synthesis [32]. Moreover, by acting as a scaffolding protein for NR and several key players of cell cycle progression and kinases, including EGFR/HER2 [33], PELP1 can mediate hormonal signaling crosstalk with cell cycle machinery [34]. Also, PELP1 interacts with STAT3 to promote its transactivation; thus potentiating the growth factor signaling axis [35]. Interestingly, PELP1 was shown to modulate expression of several genes involved in the EMT process [31]. More recently, it has demonstrated to interact with AIB1 (amplified in breast cancer 1) to enhance cancer stem cell properties [36]. Both processes were linked to basal like phenotype in breast cancer.

In summary, we demonstrated that PELP1 could be a sen- sitive marker for breast cancers, regardless of breast cancer subtypes and compared with other breast markers. However, its substantial expression level in lung/ ovarian cancers could hamper its utility in differentiating cancers of these origins. PELP1 expression is an independent adverse prognostic factor for non-luminal cancers and was associated with luminal B cancers. Interestingly, PELP1 refines the prognostic value of AR in both luminal and non-luminal cancers. The results may suggest the significance of co-regulator in prognostication of NR. These findings merit further investigation so the potential mechanistic actions and clinical value of PELP1 could be fully explored.

\section{Electronic Supplementary Material}

Supplementary materials are available at Cancer Research and Treatment website (https://www.e-crt.org).

\section{Conflicts of Interest}

Conflict of interest relevant to this article was not reported.

\section{Acknowledgments}

We would like to thank Karen Fung, Yan Shao and Ivan Poon for their technical assistance.

\section{References}

1. Chakravarty D, Tekmal RR, Vadlamudi RK. PELP1: a novel therapeutic target for hormonal cancers. IUBMB Life. 2010;62: 162-9.

2. Girard BJ, Daniel AR, Lange CA, Ostrander JH. PELP1: a review of PELP1 interactions, signaling, and biology. Mol Cell Endocrinol. 2014;382:642-51.

3. Vadlamudi RK, Manavathi B, Balasenthil S, Nair SS, Yang Z, Sahin AA, et al. Functional implications of altered subcellular localization of PELP1 in breast cancer cells. Cancer Res. 2005;65:7724-32.

4. Mann M, Zou Y, Chen Y, Brann D, Vadlamudi R. PELP1 oncogenic functions involve alternative splicing via PRMT6. Mol Oncol. 2014;8:389-400.

5. Rajhans R, Nair S, Holden AH, Kumar R, Tekmal RR, Vadlamudi RK. Oncogenic potential of the nuclear receptor coregulator proline-, glutamic acid-, leucine-rich protein $1 /$ modulator of the nongenomic actions of the estrogen receptor. Cancer Res. 2007;67:5505-12.

6. Cortez V, Samayoa C, Zamora A, Martinez L, Tekmal RR, Vadlamudi RK. PELP1 overexpression in the mouse mammary gland results in the development of hyperplasia and car- cinoma. Cancer Res. 2014;74:7395-405.

7. Brann DW, Zhang QG, Wang RM, Mahesh VB, Vadlamudi RK. PELP1: a novel estrogen receptor-interacting protein. Mol Cell Endocrinol. 2008;290:2-7.

8. Kumar R, Zhang H, Holm C, Vadlamudi RK, Landberg G, Rayala SK. Extranuclear coactivator signaling confers insensitivity to tamoxifen. Clin Cancer Res. 2009;15:4123-30.

9. Habashy HO, Powe DG, Rakha EA, Ball G, Macmillan RD, Green AR, et al. The prognostic significance of PELP1 expression in invasive breast cancer with emphasis on the ER-positive luminal-like subtype. Breast Cancer Res Treat. 2010;120: 603-12.

10. Zhang Y, Dai J, McNamara KM, Bai B, Shi M, Chan MS, et al. Prognostic significance of proline, glutamic acid, leucine rich protein 1 (PELP1) in triple-negative breast cancer: a retrospective study on 129 cases. BMC Cancer. 2015;15:699.

11. Dang DN, Raj G, Sarode V, Molberg KH, Vadlamudi RK, Peng Y. Significantly increased PELP1 protein expression in primary and metastatic triple-negative breast carcinoma: comparison with GATA3 expression and PELP1's potential role in triple-negative breast carcinoma. Hum Pathol. 2015;46:1829- 
35.

12. Vadlamudi RK, Balasenthil S, Broaddus RR, Gustafsson JA, Kumar R. Deregulation of estrogen receptor coactivator proline-, glutamic acid-, and leucine-rich protein-1/modulator of nongenomic activity of estrogen receptor in human endometrial tumors. J Clin Endocrinol Metab. 2004;89:6130-8.

13. Aust S, Horak P, Pils D, Pils S, Grimm C, Horvat R, et al. The prognostic value of estrogen receptor beta and proline-, glutamic acid- and leucine-rich protein 1 (PELP1) expression in ovarian cancer. BMC Cancer. 2013;13:115.

14. Nair SS, Guo Z, Mueller JM, Koochekpour S, Qiu Y, Tekmal RR, et al. Proline-, glutamic acid-, and leucine-rich protein$1 /$ modulator of nongenomic activity of estrogen receptor enhances androgen receptor functions through LIM-only coactivator, four-and-a-half LIM-only protein 2. Mol Endocrinol. 2007;21:613-24.

15. Slowikowski BK, Galecki B, Dyszkiewicz W, Jagodzinski PP. Increased expression of proline-, glutamic acid- and leucinerich protein PELP1 in non-small cell lung cancer. Biomed Pharmacother. 2015;73:97-101.

16. Ning Z, Zhang $\mathrm{Y}$, Chen $\mathrm{H}, \mathrm{Wu}$ J, Song $\mathrm{T}$, Wu Q, et al. PELP1 suppression inhibits colorectal cancer through c-Src downregulation. Oxid Med Cell Longev. 2014;2014:193523.

17. Elston CW, Ellis IO. Pathological prognostic factors in breast cancer. I. The value of histological grade in breast cancer: experience from a large study with long-term follow-up. Histopathology. 1991;19:403-10.

18. Lakhani SR, Ellis IO, Schnitee SJ, Tan PH, van de Vijver MJ. WHO classification of tumours of the breast. 4th ed. Lyon: IARC Press; 2012.

19. Tsang JY, Au WL, Lo KY, Ni YB, Hlaing T, Hu J, et al. PD-L1 expression and tumor infiltrating PD-1+ lymphocytes associated with outcome in HER2+ breast cancer patients. Breast Cancer Res Treat. 2017;162:19-30.

20. Tsang JY, Ni YB, Chan SK, Shao MM, Law BK, Tan PH, et al. Androgen receptor expression shows distinctive significance in ER positive and negative breast cancers. Ann Surg Oncol. 2014;21:2218-28.

21. Goldhirsch A, Winer EP, Coates AS, Gelber RD, Piccart-Gebhart M, Thurlimann B, et al. Personalizing the treatment of women with early breast cancer: highlights of the St Gallen International Expert Consensus on the Primary Therapy of Early Breast Cancer 2013. Ann Oncol. 2013;24:2206-23.

22. Nielsen TO, Hsu FD, Jensen K, Cheang M, Karaca G, Hu Z, et al. Immunohistochemical and clinical characterization of the basal-like subtype of invasive breast carcinoma. Clin Cancer Res. 2004;10:5367-74.

23. Dimple C, Nair SS, Rajhans R, Pitcheswara PR, Liu J, Balasenthil $S$, et al. Role of PELP1/MNAR signaling in ovarian tumorigenesis. Cancer Res. 2008;68:4902-9.
24. Yao T, Wang Q, Zhang W, Bian A, Zhang J. Identification of genes associated with renal cell carcinoma using gene expression profiling analysis. Oncol Lett. 2016;12:73-8.

25. Grivas PD, Tzelepi V, Sotiropoulou-Bonikou G, Kefalopoulou Z, Papavassiliou AG, Kalofonos H. Expression of ERalpha, ERbeta and co-regulator PELP1/MNAR in colorectal cancer: prognostic significance and clinicopathologic correlations. Cell Oncol. 2009;31:235-47.

26. Nishimura R, Osako T, Nishiyama Y, Tashima R, Nakano M, Fujisue $M$, et al. Evaluation of factors related to late recurrence-later than 10 years after the initial treatment-in primary breast cancer. Oncology. 2013;85:100-10.

27. Tang D, Xu S, Zhang Q, Zhao W. The expression and clinical significance of the androgen receptor and E-cadherin in triplenegative breast cancer. Med Oncol. 2012;29:526-33.

28. Peters AA, Buchanan G, Ricciardelli C, Bianco-Miotto T, Centenera MM, Harris JM, et al. Androgen receptor inhibits estrogen receptor-alpha activity and is prognostic in breast cancer. Cancer Res. 2009;69:6131-40.

29. Yang L, Ravindranathan $P$, Ramanan M, Kapur $P$, Hammes SR, Hsieh JT, et al. Central role for PELP1 in nonandrogenic activation of the androgen receptor in prostate cancer. Mol Endocrinol. 2012;26:550-61.

30. Nair SS, Mishra SK, Yang Z, Balasenthil S, Kumar R, Vadlamudi RK. Potential role of a novel transcriptional coactivator PELP1 in histone H1 displacement in cancer cells. Cancer Res. 2004;64:6416-23.

31. Roy S, Chakravarty D, Cortez V, De Mukhopadhyay K, Bandyopadhyay A, Ahn JM, et al. Significance of PELP1 in ER-negative breast cancer metastasis. Mol Cancer Res. 2012;10: 25-33.

32. Rajhans R, Nair HB, Nair SS, Cortez V, Ikuko K, Kirma NB, et al. Modulation of in situ estrogen synthesis by proline-, glutamic acid-, and leucine-rich protein-1: potential estrogen receptor autocrine signaling loop in breast cancer cells. Mol Endocrinol. 2008;22:649-64.

33. Vadlamudi RK, Kumar R. Functional and biological properties of the nuclear receptor coregulator PELP1/MNAR. Nucl Recept Signal. 2007;5:e004.

34. Sareddy GR, Vadlamudi RK. PELP1: Structure, biological function and clinical significance. Gene. 2016;585:128-34.

35. Manavathi B, Nair SS, Wang RA, Kumar R, Vadlamudi RK. Proline-, glutamic acid-, and leucine-rich protein-1 is essential in growth factor regulation of signal transducers and activators of transcription 3 activation. Cancer Res. 2005;65:5571-7.

36. Truong TH, Hu H, Temiz NA, Hagen KM, Girard BJ, Brady NJ, et al. Cancer stem cell phenotypes in $\mathrm{ER}(+)$ breast cancer models are promoted by PELP1/ AIB1 complexes. Mol Cancer Res. 2018;16:707-19. 